\title{
Comparison of Vitamin D Status Between Urban and Rural South Indian Mothers and Their Newborns
}

\author{
Kumar KJ1', Chavan $A^{2}$, Shushma $K^{3}$, Murthy $S^{4}$
}

${ }^{1}$ Dr. K Jagadish Kumar, MBBS. MD, Professor of Paediatrics, JSS Medical College, JSS University, Mysore, India, ${ }^{2} \mathrm{Dr}$. Abhishek Chavan, MBBS. MD, Fellow in Paediatric Critical Care, KEM Hospital and Research Centre, Pune, India, ${ }^{3}$ Dr. Sushma K, MBBS. MD, Assistant Professor, Department of Paediatrics, JSS Medical College, JSS University, Mysore, India. ${ }^{4} \mathrm{Dr}$.Srinivasa Murthy D. MBBS. MD, Associate Professor, Department of Paediatrics, JSS Medical College, JSS University, Mysore,India.

Address for correspondence:

Dr. K. Jagadish Kumar

85/B, 9TH Cross, Navilu Road,

Kuvempu Nagar, Mysore,

Karnataka, India-570023

Tel: +919844281859

E-mail: jagdishmandya@gmail.com

Acknowledgements: None

Funding: Nil

Conflict of Interest: None

Permission from IRB: Yes

Ethical dilemmas faced during study: No

\section{How to cite}

Kumar KJ, Chavan A, Shushma K. Murthy S. Comparison of Vitamin D Status Between Urban and Rural South Indian Mothers and Their Newborns. J Nepal Paediatr Soc 2016;36(3):243-249.

doi: http://dx.doi.org/10.3126/jnps.v36i3.15156

This work is licensed under a Creative Commons Attribution 3.0 License.

\begin{abstract}
Introduction: Though pregnant mothers are at risk of developing Vitamin D deficiency, their level differs among the urban and rural population and has various implications on newborns. This study was undertaken to compare the vitamin D status in urban and rural population of South India among pregnant women and their newborns.Material and Methods:Ninety one pregnant women and their newborns (46 urban and 45 rural) were recruited. Maternal Vitamin D levels and serum concentrations of calcium, phosphorus, alkaline phosphatase and Vitamin D were measured in the cord blood. Results:Eighty two $(90 \%)$ of the pregnant women, 38 from rural and 44 from urban, had vitamin D deficiency. However, the mean vitamin $D$ levels were low in urban $(9.42 \pm 12.2)$ compared to rural women $(35.86 \pm 16.3, p=0.037)$. Hypovitaminosis $D$ was noted in $88 \%$ of the newborns, deficiency being more in neonates born to urban women. Though the dietary calcium intake was uniformly low, it was lower in rural $(960.1 \pm 139.9$ $\mathrm{mg} / \mathrm{d})$ Vs urban mothers $(1060.1 \pm 150.6 \mathrm{mg} / \mathrm{d}, p<0.001)$ Urban mothers were found to be exposed to sun for lower duration of 0.3 hours compared to rural mothers 0.5 hours. Similarly the surface area of body exposed to sun was lower in urban mothers $(7.5 \%)$ compared to rural mothers $(15 \%$, $p=0.0001$ ). A moderate positive correlation between motherneonate pair was noted. Conclusions:The magnitude of Hypovitaminosis D observed among pregnant women and their newborn warrants public health intervention in terms of vitamin D supplementations during pregnancy.
\end{abstract}

Key words: Vitamin D Deficiency, Pregnancy, Rural and Urban women, Sun exposure, Newborns.

\section{Introduction}

$\mathrm{V}$ itamin D deficiency related illness is now an emerging global threat. Currently, there is increased recognition of various hitherto unrecognized functions of Vitamin $D$ resulting in it being recognized as hormone. This has led to implication of Vitamin D deficiency as cause of various illness ranging from cardiovascular to immunological ${ }^{1,2,3,4}$. Evidences have disproved the earlier belief that Vitamin $D$ deficiency is rare disease in countries with abundant sunshine and there is 
increasing evidence to show that vitamin $D$ deficiency is highly prevalent in India,6,7. Cultural practices of remaining indoor, higher skin pigmentation and poverty leading to poor intake may all be contributory. As such the daily dietary intake of calcium of both the urban and rural population is low compared to that of recommended daily/dietary allowances (RDA) issued by Indian Council of Medical Research ${ }^{8,9}$.Current supplementation recommendations are inadequate to ensure vitamin $D$ sufficiency in pregnant and lactating women ${ }^{10}$. Sahuet al reported a high prevalence of vitamin $D$ deficiency among adolescent girls and pregnant women from a rural Indian community. However boys were relatively protected ${ }^{5}$. Pregnant mothers are at higher risk of developing Vitamin D deficiency due their increased needs which is compounded by poor intake particularly in developing countries like India. Trans placental transfer is the main source of Vitamin D in the newborns ${ }^{6,11}$. Hence Vitamin $\mathrm{D}$ deficiency during pregnancy may result in immediate newborn illness such as hypocalcaemic seizures and poor Vitamin D stores ${ }^{6,12}$.Further, this leads to Vitamin $D$ deficiency in breastfed infants ${ }^{10}$. These babies are susceptible to lower respiratory infections which contribute to increased infant mortality rate ${ }^{6,13}$. The prevalence of Vitamin $D$ deficiency in pregnant mothers in India, is alarming ${ }^{5,6,7}$. Hypovitaminosis $D$ is prevalent among both rural and urban population. However due to difference in environment, life style and nutritional status, the magnitude of this problem varies between these two populations groups and in some studies urban subjects are found to be more deficient ${ }^{5,6,14,15,16}$.Studies looking into the Vitamin D levels of mother-newborn pair and comparing this between rural and urban population is scare. Hence this study was undertaken to compare to the Vitamin $\mathrm{D}$ levels between urban and rural mothers and their respective newborns.

The primary objective of this study was to compare the vitamin $D$ status between pregnant women from urban and rural populations. The secondary objectives were to compare the levels of vitamin $D$ in the cord blood of urban and rural mothers and also to study the correlation between vitamin $\mathrm{D}$ levels of mother and their newborn.

\section{Material and Methods}

This study was conducted at JSS hospital Mysuru from August 2013 to December 2013. JSS hospital is a tertiary care hospital with approximately 3000 deliveries per year and care for high risk pregnant mothers and this hospital also offers tertiary level neonatal care. Singleton pregnant mothers admitted for delivery and their newborns were eligible for this study.Patients with chronic liver disease, renal disease, diabetes, preeclampsia and on medications such as anti-tubercular, anti-epileptic drugs or steroids within the last 3 months were excluded. Mothers were classified to be from Urban or Rural area as per the definition of Ministry of Home Affairs, Government of India ${ }^{17}$.

This was a prospective observational analytical study. The mothers included were interviewed for their pregnancy status, diet, occupation, pattern of clothing, supplemental calcium and history suggestive of osteomalacia such as proximal muscle weakness, bone pain or tenderness and recurrent fractures. History of the daily out door work and the type of clothing worn taken to assess the sunshine exposure and was calculated as hours of exposure/day $x$ percentage of body surface area (BSA) exposed. Calcium intake in the diet was assessed by 24 hour recall method and calculated as per the ICMR $s$ charts for nutritive values of Indian food. Three $\mathrm{ml}$ of maternal and cord blood was sent for analysis of Vitamin D [25(OH) D ] by chemilumiescence assay.Cord blood was also analysed for calcium and Alkaline phosphatase.

Estimation of sample size was based on the mean and standard deviation, reported by a study from northern India. In order to estimate the target difference of vitamin $D$ levels between urban and rural mothers by $5 \%$, with an alpha error of 0.05 and beta error of 0.2 , the required sample size was calculated to be a minimum of 45 from urban and rural each. We recruited a total of 91 consecutive pregnant women and their newborns from both urban and rural regions in our study.

Statistical analysis was done using SPSS v16.0 software. Continuous variables were presented as mean \pm SD. Proportions were compared by using the chi-square test or Fischer exact test as appropriate. Correlations were studied by using Pearson product moment correlation and $p$-value of $<0.05$ was considered as statistically significant.

Informed written consent was taken from all patients that were included. Ethical clearance was obtained from the institutional ethical committee of JSS medical college.

\section{Results}

Hypovitaminosis D was observed in 82 out of 91 pregnant women enrolled in the study, with a prevalence rate of $90 \%$. Only three women had sufficient Vitamin D levels and all of them were from rural area. However the 
difference in prevalence was not statistically significant $(p=0.09)$.

The mean serum Vitamin $D$ levels in the pregnant women from urban regions (29.42 \pm 12.2) was significantly lower compared to their rural counterparts (35.86 \pm 16.3) Table 2 .

Only $4(4.3 \%)$ of the cord blood studied were Vitamin D sufficient, 7 (7.7\%) samples had vitamin D insufficiency and 80 (88\%) had vitamin D deficiency [Table 3]. We also found a higher percentage of cord blood Vitamin $D$ level deficiencies in neonates born to urban mothers compared to the neonates of rural mothers, i.e. $91 \%$ of urban vs $84 \%$ of rural cord blood samples $(p=0.35)$ Table 3.

On comparing the mean cord blood vitamin $D$ levels in urban $(33.61 \pm 15.2)$ and rural $(38.37 \pm 18.3)$ neonates, we found the difference was not statistically significant [Table 2]. On assessing the calcium intake by 24 hour recall and calculated by ICMR charts, it was observed that both dietary as well as supplemental calcium intake was uniformly low in both groups, with calcium intake being significantly higher in urban women $(1060.1 \pm 150.6 \mathrm{mg} / \mathrm{d})$ compared to rural women $(960.1$ $\pm 139.9 \mathrm{mg} / \mathrm{d}, p<0.001)$. However mean cord blood calcium levels of neonates born to urban mothers was significantly lower compared to their rural counterparts. Cord blood alkaline phosphatase levels were more in urban mothers. There were 9 mothers belonging to Muslim religion ( 2 of them from rural and 7 from urban) and all of them were found to be Vitamin $D$ deficient.

Urban mothers were found to be exposed to sun for lower duration of 0.3 hours (Median) compared to rural mothers 0.5 hours (Median).Similarly the surface area of body exposed to sun was lower in urban mothers $(7.5 \%)$ compared to rural mothers $(15 \%, p<0.0001)$ Table 4.

Mother - neonate pair showed a moderate correlation of $67.2 \%$ with respect to Vitamin $D$ levels in rural population. There was a low correlation of $48 \%$ in mother - neonate pair from urban area (Figure $1 \& 2$ ).

Table 1: Comparison of Vitamin D Status between urban and rural pregnant women

\begin{tabular}{|c|c|c|c|c|c|}
\hline \multirow[b]{2}{*}{ Vitamin D levels [25(OH) D] } & \multicolumn{3}{|c|}{ Number of mothers } & \multirow[b]{2}{*}{$x^{2}$ value } & \multirow[b]{2}{*}{$p$-value * } \\
\hline & $\begin{array}{c}\text { Total } \\
\mathrm{n}=91\end{array}$ & $\begin{array}{l}\text { Urban } \\
n=46\end{array}$ & $\begin{array}{l}\text { Rural } \\
n=45\end{array}$ & & \\
\hline Sufficient [>75 nmol/l] & $3(3.2 \%)$ & 0 & $3(7 \%)$ & 1.4 & 0.12 \\
\hline Insufficient [50-75 nmol /I] & $6(6.5 \%)$ & $2(4 \%)$ & $4(9 \%)$ & 0.21 & 0.43 \\
\hline Deficient $\quad[<50 \mathrm{nmol} / \mathrm{l}]$ & $82(90.1 \%)$ & $44(96 \%)$ & $38(84 \%)$ & 2.1 & 0.09 \\
\hline
\end{tabular}

* Chi square test

Table 2: Comparison of Maternal and Neonatal Vitamin D, Calcium and alkaline phosphatase levels between Urban and Rural areas

\begin{tabular}{|c|c|c|c|c|c|c|}
\hline & Urban & Rural & $\begin{array}{c}\text { Mean } \\
\text { Diff }\end{array}$ & & & \\
\hline & Mean (SD) & Mean (SD) & & $\mathbf{t}$ & $\mathrm{Cl}$ & p \\
\hline Maternal Vitamin D [nmol/l] & $29.4(12.29)$ & $35.8(16.3)$ & 6.43 & 2.11 & 0.39 to 12.4 & 0.037 \\
\hline Maternal Calcium intake[mg] & $1060.1(150.57)$ & $901(139.9)$ & -159.1 & 5.22 & -219.4 to -98.5 & 0.0001 \\
\hline $\begin{array}{l}\text { Cord blood } \\
\text { Vitamin D [nmol/l] }\end{array}$ & $33.6(15.2)$ & $38.3(18.2)$ & 4.75 & 1.33 & -2.28 to 11.6 & 0.18 \\
\hline Cord blood Calcium [mg/DI] & $9.5(1.07)$ & $10.0(1.1)$ & 0.51 & 2.13 & 0.03 to 0.96 & 0.035 \\
\hline Cord Blood Alkaline phosphatase [ U/L] & $562(360.5)$ & $442.8(540.9)$ & 0.14 & & 0.0005 to 0.28 & 0.05 \\
\hline
\end{tabular}

Table 3: Comparison of Vitamin D Status between Neonates born to urban and rural pregnant mothers

\begin{tabular}{|c|c|c|c|c|c|}
\hline \multirow{3}{*}{ Cord blood Vitamin D levels [25(OH) D] } & \multicolumn{3}{|c|}{ Number of newborns } & \multirow{3}{*}{$\mathrm{X}^{2}$ value } & \multirow{3}{*}{$p$-Value * } \\
\hline & Total & Urban & Rural & & \\
\hline & $\mathbf{n}=91$ & $n=46$ & $n=45$ & & \\
\hline Sufficient $[>75 \mathrm{nmol} / \mathrm{l}]$ & $4(4.3 \%)$ & $1(2 \%)$ & $3(7 \%)$ & 0.28 & 0.36 \\
\hline Insufficient [50-75 nmol /l] & $7(7.7 \%)$ & $3(7 \%)$ & $4(9 \%)$ & 0.0009 & 0.71 \\
\hline Deficient [<50 nmol/l ] & $80(88 \%)$ & $42(91 \%)$ & $38(84 \%)$ & 0.46 & 0.35 \\
\hline
\end{tabular}

${ }^{*}$ Chi square test 
Table 4: Comparison of Sunlight exposure between Rural and Urban Mothers

\begin{tabular}{lcccccccccc}
\hline & \multicolumn{3}{c}{ Total } & \multicolumn{3}{c}{ Urban } & \multicolumn{3}{c}{ Rural } & \multicolumn{1}{c}{ p-value* $^{*}$} \\
\cline { 2 - 11 } & Median & Q1 & Q3 & Median & Q1 & Q3 & Median & Q1 & Q3 & \\
\hline Sun exposure time (hours/day) & 0.5 & 0.25 & 0.5 & 0.3 & 0.25 & 0.5 & 0.5 & 0.5 & 0.5 & 0.0001 \\
$\begin{array}{l}\text { Sun Exposure } \\
\text { (\% BSA) }\end{array}$ & 15 & 7.5 & 15 & 7.5 & 5 & 10 & 15 & 15 & 20 & 0.0001 \\
\hline
\end{tabular}

*Mann Whitney $U$ test, $\mathrm{Q} 1=1^{\text {st }}$ quartile, $\mathrm{Q} 3=3^{\text {rd }}$ quartile, BSA = Body surface area

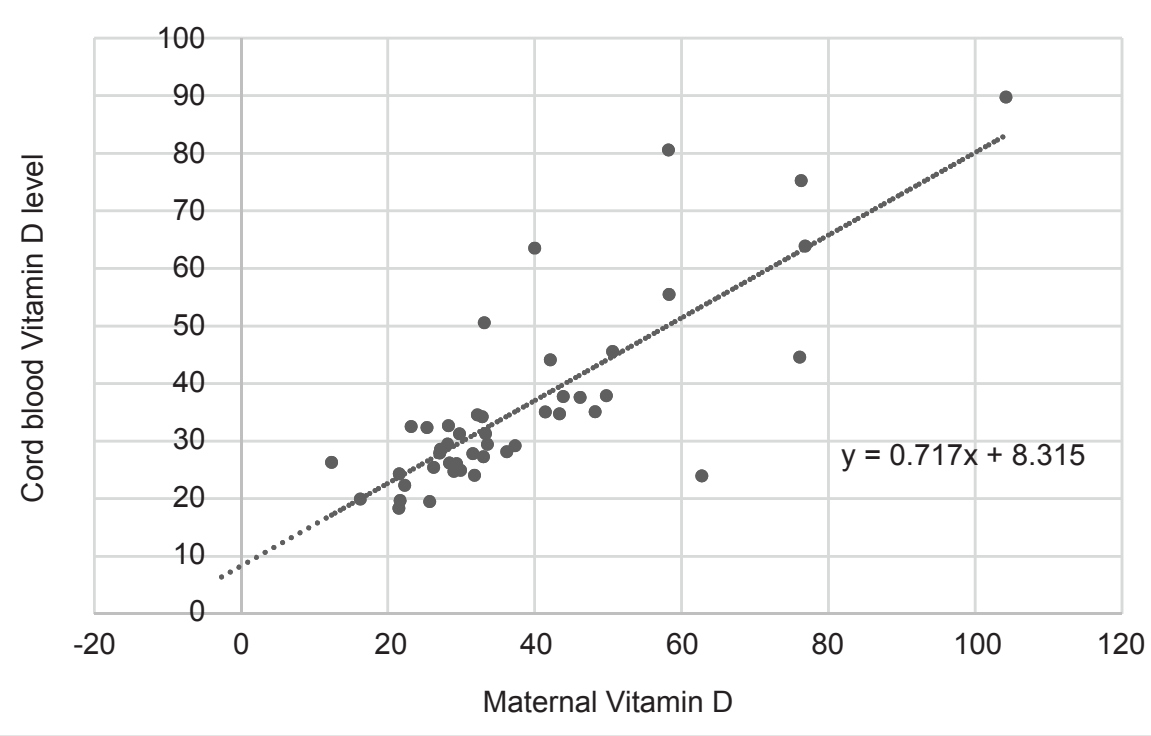

Fig 1: Correlation between maternal and cord blood Vitamin D level in rural mother-neonate pair

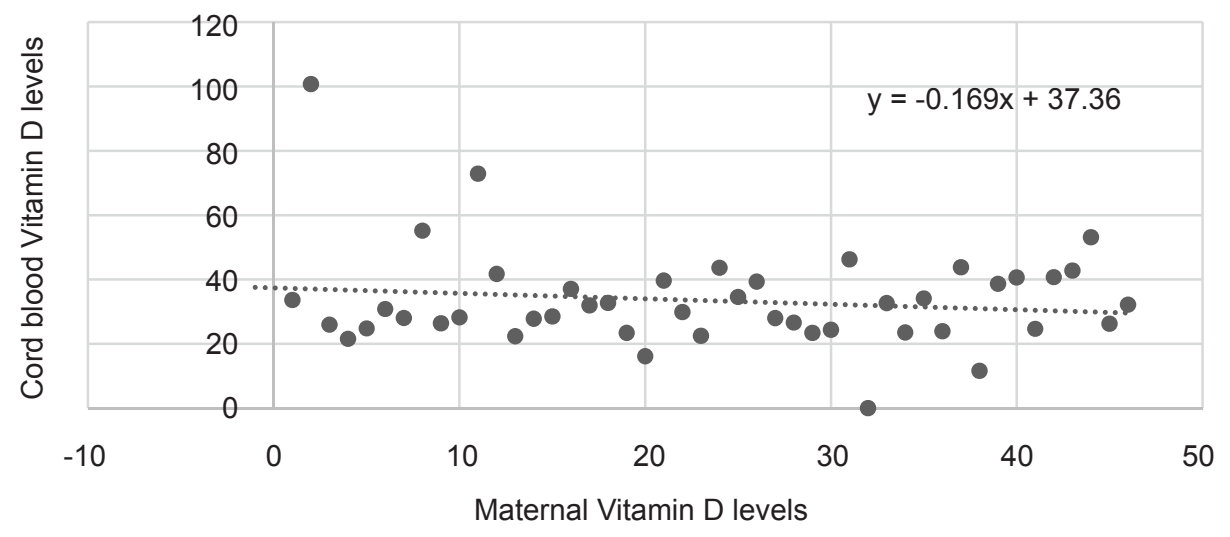

Fig 2: Correlation between maternal and cord blood Vitamin D level in urban mother-neonate pair

\section{Discussion}

Vitamin D deficiency has become a worldwide phenomenon, as it is prevalent in most of the developing countries in all age groups residing in both rural and urban areas ${ }^{18}$. Pregnant mothers are at higher risk of developing Vitamin $D$ deficiency due their increased needs andpoor intake and has been reported from most of the developing countries ${ }^{7,14,19,20,21}$. The prevalence of Vitamin D deficiency in pregnant mothers in India, in spite of it being a tropical country is alarming $5,6,7$. Presently, vitamin D supplementation is not a part of antenatal care programs in India ${ }^{6}$. The United States Institute of Medicine has recently defined levels of serum 25(OH)D greater than $50 \mathrm{nmol} / \mathrm{L}($ or $20 \mathrm{ng} / \mathrm{mL}$ ) as adequate for pregnant women ${ }^{22}$.Study by Holmes et al revealed that vitamin $\mathrm{D}$ concentrations were lower in pregnant women compared to non-pregnant women ${ }^{3}$. 
Our study, further support this fact and found that $90 \%$ of pregnant women had Vitamin D deficiency. Studies by Sachan et al and Marwaha et al observed that $84 \%$ and $96.3 \%$ of pregnant women to be vitamin D deficient respectively $^{6,7}$. But Dasgupta et al reported relatively lower percentage $(42 \%)$ of vitamin D deficiency among pregnant women as compared to the other Indian studies $^{23}$. None of the pregnant women had adequate vitamin $D$ concentrations ( $\geq 30.0 \mathrm{ng} / \mathrm{ml}$ ) in a study from India ${ }^{13}$. Similar trend of high prevalence of Vitamin D deficiency in pregnant mothers has been reported from other countries as well11,12,14,19,20,21. Hariharan et al and Goswamiet al revealed that vitamin D levels of rural adult subjects were significantly higher than that of urban adult subjects in both males and females ${ }^{16,17}$. The mean Vitamin $D$ levels in the serum were significantly low in urban mothers compared to rural mothers. However it is interesting to see that there was no statistically significant difference in the prevalence of hypovitaminosis $D$ per se, between urban and rural pregnant mothers. Nurbazlinet al from Malaysia also noted that vitamin $D$ levels of rural women was significantly higher [69.5 (59.0-79.1) $\mathrm{nmol} / \mathrm{L}]$ compared to urban women [31.9 (26.1- 45.5) $\mathrm{nmol} / \mathrm{L}](p<0.001)$. In contrast to our study, most of their study population from rural area did not have Vitamin $D$ deficiency ${ }^{14}$.This phenomenon does not seem to be common in all geographies. Sachan et al from north India, showed the mean serum $25(\mathrm{OH}) \mathrm{D}$ concentration in urban women did not differ significantly from that in rural women ${ }^{6}$. Furthermore, in China, a study by Wang et al observed that the mean vitamin $D$ levels were significantly lower $(19.0 \pm 6.75 \mathrm{nmol} / \mathrm{l})$ in rural pregnant women as compared to the urban mothers $(48.52 \pm 16.48$ $\mathrm{nmol} / \mathrm{l}, p<0.05)$; they attributed this to the lower calcium intake in the rural pregnant women compared to their urban counterparts ${ }^{11}$. In our study, the high prevalence of Vitamin $D$ deficiency in urban women mothers as compared to their rural counterparts may be attributed to inadequate sunlight exposure, traditional clothing, and occupation, as most of them work indoors in salwar compared to rural women working in fields in saree. Sun exposure is the major source of vitamin $D$ and plays an important role in maintaining normal vitamin $D$ levels ${ }^{2,4,24}$.A similar study from Malaysiaalso observed that the majority of urban women $(43.9 \%)$ were vitamin $\mathrm{D}$ deficient, whilst the majority of rural women $(88.1 \%)$ were vitamin $\mathrm{D}$ sufficient and they explained that rural women spent significantly more time under the sun compared to urban women14. A study from urban area of China suggested that the pregnant women working indoors are at high risk of vitamin D insufficiency ${ }^{25}$.
We observed that the dietary intake of calcium was uniformly low, and it was significantly more so in rural than in urban women. This could be explained by the higher social economic status of urban mothers. Similar observation was made by other authors ${ }^{6,11,14}$. Harharan et al found out that dietary calcium was significantly lower in both the rural adult and children compared to that of the urban adult and children ${ }^{8}$. There were nine mothers in our study, belonging to Muslim religion (two from rural and seven from urban) and all of them were Vitamin $D$ deficient, which can be explained by the cultural practice of wearing purdah. A study from Turkey also revealed that Vitamin $D$ concentrations of mothers with traditional Islamic dress which covers entire body including face and hands was significantly lower compared with mothers who wore conventional dress which exposed face and hands ${ }^{21}$

Vitamin D levels depends on various factors like occupation, clothing, skin pigmentation, sunlight exposure,use of sunscreen, nutrition and supplements ${ }^{18,26}$. Hypovitaminosis D may be attributed to inadequate sunlight exposure, indoor occupation, traditional clothing along with a low dietary calcium intake $e^{2,3,4}$. This is further compounded by air pollution, and decreased outdoor activity as a result of urbanization.

Jani et al study revealed that sun exposure index was positively associated with serum Vitamin $\mathrm{D}$ concentrations and it remained a significant and a considerable independent variable. Both duration of exposure to sunlight and the body surface area exposed is important for adequate vitamin $D$ synthesis ${ }^{13}$.Skin pigmentation, duration of sun exposure and the body surface area exposed to sun light all could explain the findings of lower levels of vitamin D in urban mothers found in our study.

Cord blood hypovitaminosis D (88\%) observed in our study is significantly high and the results are similar to other studies ${ }^{6,11,12,24}$.Despite there being a difference in maternal levels, both urban and rural neonates were having a uniformly low mean vitamin $D$ levels with no significant difference. Sachan et al study from North India revealed a large proportion of neonates (95.7\%) had hypovitaminosis $D^{6}$. However in a study from China,the mean level of serum Vitamin $D$ in urban neonates was significantly more than that in rural neonates ${ }^{11}$.

Several studies have shown a positive correlation between maternal vitamin $\mathrm{D}$ levels and cord blood vitamin $D$ levels ${ }^{7,11,12,24,27-29}$. We observed a dichotomous phenomenon where the correlation between rural 
mother-neonate pair was moderate $(67.2 \%)$ whereas that in the urban mother-neonate pair was poor (48\%). Despite there being a difference in maternal levels, both urban and rural neonates had a uniformly low mean vitamin $D$ levels with no significant difference.This indicates the possibility of several other yet undetermined factors influencing the fetal level of Vitamin D apart from the maternal Vitamin D levels.

We observed that the cord blood calcium levels which is significantly lower in urban newborns compared to their rural counterpart andAlkaline phosphatase(ALP) levels which were high in urban newborns when compared to the rural newborns. Similar observation was made by Maghbooliet al also' ${ }^{12}$.

Consequent to this study and literature search, we believe it is important to redefine the cut off levels of Vitamin $D$ deficiency status. Such a high prevalence of Vitamin $D$ deficiency status in our study and several other studies across the globe indicate the need for better representative cutoff value. Recommendation of different cut-off values by various agencies itself suggests the limitation of using any one cut-off value. Secondly, is the issue of normal value of Vitamin $D$ levels in the newborn. We suspect that this question will remain unsettled unless one physiologically relevant cut off value in the mother is defined.Third are the factors influencing the levels of Vitamin D level in the fetus? Further research in this field would be able to provide better insight into the Vitamin D status in newborns.

\section{Conclusion}

Our study shows that the prevalence of Vitamin $D$ deficiency in pregnant mothers is high and there is no significant difference between urban and rural populations. Rural mothers have higher levels of serum Vitamin D compared to urban mothers which indicate the complex interplay of factors such as skin pigmentation, duration of sun exposure, body surface area exposed to sun and other cultural, occupational and economic factors.

Cord blood Vitamin D levels is at best has only moderate positive correlation with maternal vitamin $D$ levels.

Limitations of the Study: Maternal vitamin D levels were not correlated with that of parathyroid harmone PTH and ALP levels.

\section{References}

1. Goswami S.K., Mishra, Kochupillai. N. Prevalence \& potential significance of vitamin $D$ deficiency in Asian Indians. Indian J Med Res 2008;127:229-38.

2. Goswami R, Gupta N, Goswami D, et al. Prevalence and significance of low 25-hydroxyvitamin D concentrations in healthy subjects in Delhi.Am J Clin Nutr 2000;72:472-75.

3. Holmes VA, Barnes MS, Alexander HD, et al.Vitamin $\mathrm{D}$ deficiency and insufficiency in pregnant women: a longitudinal study. Br J Nutr 2009;102:876-81.

4. Dawodu A, Wagner CL. Mother child vitamin D deficiency: an international perspective.Arch Dis Child 2007;92:737-40

5. Sahu M, Bhatia V, Aggarwal A, et al. Vitamin D deficiency in rural girls and pregnant women despite abundant sunshine in northern India.Clin Endocrinol (Oxf) 2009;70:680-684.

6. Sachan A, Gupta R, Das V, et al. High prevalence of vitamin $\mathrm{D}$ deficiency among pregnant women and their newborns in northern India. Am J Clin Nutr 2005;81:1060-64.

7. Marwaha K, Tandon N, Chopra S,et al. Vitamin D status in pregnant Indian women across trimesters and different seasons and its correlation with neonatal serum 25-hydroxyvitamin D levels. Br J Nutr 2011;106:1383-389.

8. Harinarayan C V, Ramalakshmi T, Prasad U V., et al. Vitamin D status in Andhra Pradesh : A population based study. Indian J Med Res2008;127:211-18.

9. Kaushal M, Magon N. Vitamin $D$ in pregnancy: A metabolic outlook. Indian J Endocrinol Metab 2013;17:76-82.

10. Balasubramanian S. Vitamin $D$ deficiency in breastfed infants \& the need for routine vitamin D supplementation. Indian J Med Res 2011;133:250-52.

11. Wang J, Yang F, Mao M., et al.High prevalence of vitamin $\mathrm{D}$ and calcium deficiency among pregnant women and their newborns in Chengdu, China. World J Pediatr 2010;6:265-67.

12. Maghbooli Z, Hossein-Nezhad A, ShafaeiAR.,etal. Vitamin D status in mothers and their newborns in Iran. BMC Pregnancy Childbirth 2007;7:1 doi:10.1186/14712393-7-1.

13. Jani R, Suhaila Palekar, Tanya Munipally., et al Widespread 25- Hydroxyvitamin D Deficiency in Affluent and Nonaffluent Pregnant Indian Women. Biomed Res Int 2014;Article ID 892162, 8 pages http:// dx.doi.org/10.1155/2014/892162. 
14. Nurbazlin M, Chee WS, Rokiah P.,et al. Effects of sun exposure on $25(\mathrm{OH})$ vitamin $\mathrm{D}$ concentration in urban and rural women in Malaysia.Asia Pac J Clin Nutr 2013;22;391-399.

15. Moy FM, Bulgiba A. High prevalence of vitamin D insufficiency and its association with obesity and metabolic syndrome among Malay adults in Kuala Lumpur, Malaysia. BMC Public Health.2011;11:735.

16. Harinarayan C V , Ramalakshmi T, Prasad U V., et al. High prevalence of low dietary calcium, high phytate consumption, and vitamin $\mathrm{D}$ deficiency in healthy south Indians. Am J Clin Nutr2007;85;1062-67.

17. Censusindia.gov.in[Internet].Office of The Registrar General \& Census Commissioner, India, New Delhi :[updated 2011, Aug 2013]Available from :http:// www.censusindia.gov.in

18. Goswami R, Kochupillai N, Gupta N,et al. Presence of $25(\mathrm{OH}) \mathrm{D}$ deficiency in a rural north Indian village despite abundant sunshine.J Assoc Physicians India 2008;56:755-757.

19. Tao M, Shao H, GuJ.,et al. Vitamin D status of pregnant women in Shanghai, China.J Matern Fetal Neonatal Med 2012;25:237-39.

20. Bassir M, Laborie S, LapilloneA,et al. Vitamin D deficiency in Iranian mothers and their neonates: a pilot study. Acta Paediatr 2001;90:577-79.

21. Alagöl $F$, Shihadeh $Y$, Boztepe $H$, et al. Sunlight exposure and vitamin $\mathrm{D}$ deficiency in Turkish women.J Endocrinol Invest 2000;23:173-77.
22. Flood-Nichols SK, Tinnemore D, Huang RR,et al. Vitamin D Deficiency in Early Pregnancy.PLoS One2015;10(4):e0123763.

23. Dasgupta A, Saikia U, Sarma D. Status of $25(\mathrm{OH}) \mathrm{D}$ levels in pregnancy: A study from the North Eastern part of India.Indian J EndocrinolMetab2012;16(suppl 2), S405-7.

24. Karim S A,Nusrut U, Aziz S. Vitamin D deficiency in pregnant women and their newborns as seen at a tertiary-care center in Karachi,Pakistan. Int $\mathrm{J}$ GynaecolObstet 2011;112:59-62.

25. Xiang $\mathrm{F}$, Jiang $\mathrm{J}$, $\mathrm{Li} \mathrm{H}$, et al. High prevalence of vitamin $D$ insufficiency in pregnant women working indoors and residing in Guiyang, China.J Endocrinol Invest2013; 36:503-7.

26. LipsP.Worldwide status of vitamin D nutrition.J Steroid Biochem Mol Biol2010; 121:297-300.

27. Lee JM, Smith J, Philipp BL,et al. Vitamin D deficiency in a healthy group of mothers and newborn infants. Clin Pediatr (Phila)2007;46:42-44

28. Nicolaidou P, Hatzistamatiou Z, Papadopoulou A, et al. Low vitamin $D$ status in mother-newborn pairs in Greece.Calcif Tissue Int 2006;78:337-42.

29. Zeghoud F, Vervel C, GuillozoH.,et al Subclinicalvitamin $D$ deficiency in neonates: definition and response to vitamin D supplements. Am J Clin Nutr 1997;65:77178. 